\title{
Molecular mechanism of the effect of angiopoietin-like protein 8 on the proliferation, invasion and migration of placental trophoblasts in preeclampsia
}

\author{
WEIQI WANG ${ }^{1}, \mathrm{XIAOQIONG} \mathrm{LI}^{2}$ and DONGLIN JI ${ }^{2}$ \\ ${ }^{1}$ Department of Obstetrics and Gynecology, Xuzhou Central Hospital, Xuzhou, Jiangsu 221009; \\ ${ }^{2}$ Department of Obstetrics and Gynecology, Huai'an Maternity and Child Health Hospital, Huai'an, \\ Jiangsu 223002, P.R. China
}

Received June 10, 2019; Accepted November 28, 2019

DOI: $10.3892 /$ etm.2020.8647

\begin{abstract}
Preeclampsia (PE) is a pregnancy-specific systemic disorder characterized by various manifestations of organ dysfunction. Inadequate trophoblastic invasion of the uterine wall is involved in the pathogenesis of PE. Angiopoietin-like protein 8 (ANGPTL8) serves an important role in cardiovascular disease development and may have a potential effect on cell proliferation. In the present study, downregulation of ANGPTL8 promoted cell proliferation, decreased p21 expression, and increased the expression levels of cyclin-dependent kinase 2 and proliferating cell nuclear antigen in HTR8/SVneo cells. Silencing of ANGPTL8 led to significant acceleration in cell migration and invasion, and markedly enhanced the matrix metalloproteinase (MMP)-2 and MMP-9 expression levels. In addition, the protein expression levels of tissue inhibitor of matrix metalloproteinase (TIMP)-1 and TIMP-2 were decreased in the group transfected with small interfering RNA (si)-ANGPTL8-1 as compared with those in the control and si-negative control groups. Taken together, these results indicated that ANGPTL8 downregulation promoted the proliferation, migration and invasion of trophoblast cells. Thus, ANGPTL8 suppresses the viability, proliferation, migration and invasion of trophoblast cells, and may be a potential therapeutic target for the clinical treatment of PE.
\end{abstract}

Correspondence to: Miss Xiaoqiong $\mathrm{Li}$ or Mr. Donglin $\mathrm{Ji}$, Department of Obstetrics and Gynecology, Huai'an Maternity and Child Health Hospital, 104 Renmin South Road, Huai'an, Jiangsu 223002, P.R. China

E-mail: lixiaoqiong2019@126.com

E-mail: hhyvivian@sina.com

Key words: preeclampsia, angiopoietin-like protein 8, trophoblast cells, proliferation, invasion, migration

\section{Introduction}

Preeclampsia (PE) is a pregnancy-specific disorder that causes considerable maternal and perinatal morbidity and mortality worldwide (1), occurring in $2-8 \%$ of pregnancies, particularly in developing countries $(2,3)$. PE is associated with acute and long-term complications, including enhanced platelet aggregation, activation of the coagulation system, endothelial dysfunction and vasospasm (4). It has been reported that women who suffered from PE during their pregnancies had a higher risk of heart failure, stroke and mortality as a result of cardiovascular disease compared with that of healthy pregnant women (5). Although the exact cause and pathophysiology of PE is yet not fully understood, it is generally considered that the placenta plays an important role in the pathogenesis of PE, since removal of the placenta can eliminate the clinical symptoms of women with PE (6). Furthermore, growing evidence indicates that abnormally shallow placentation serves a major role in the pathogenesis of PE (7). Inadequate trophoblastic invasion of the uterine wall, excessive apoptosis of trophoblast cells and impaired spiral artery transformation at the maternal-fetal interface are major abnormalities in the development of the placenta, which persistently increase resistance to blood flow in the uteroplacental circulation $(8,9)$. It has recent been reported that the incidence of PE was decreased by elective delivery, and antihypertensive drug therapy must be applied to treat severe hypertension in women with PE (10).

Angiopoietin-like proteins (ANGPTLs) comprise a class of secreted factors that are characterized by an N-terminal coiled-coil domain and a C-terminal fibrinogen-like domain, and are structurally similar to angiopoietin. However, unlike other ANGPTLs, ANGPTL8 lacks the C-terminal fibrinogen-like domain $(11,12)$. A previous study has reported that ANGPTL4 and ANGPTL8 are multifunctional factors associated with the regulation of lipid metabolism, which can bind to lipoprotein lipase (LPL) and antagonize its activity (13). Since LPL hydrolyzes triglycerides (TGs) circulating in the capillaries of adipose tissue and the muscles, resulting in increased TG concentrations in the plasma $(11,12,14,15)$. In addition, enhanced levels of circulating ANGPTL8 have been reported 
to be positively correlated with circulating low-density lipoprotein-cholesterol levels and inversely correlated with circulating high-density lipoprotein (HDL)-cholesterol levels in the serum (13), suggesting that circulating ANGPTL8 concentrations serve an important role in cardiovascular disease development. A recent study has demonstrated that ANGPTL4 mediates the protective role of activators of peroxisome proliferator-activated receptor $\gamma$ and is associated with the pathogenesis of PE (16). However, the expression and secretion of ANGPTL8 has not been investigated in the onset of PE. Recently, it was revealed that ANGPTL8 has a potential effect on cell proliferation (17). Thus, it is hypothesized that ANGPTL8 may have a potential effect on the pathogenesis of PE.

The present study aimed to investigate the effect of ANGPTL8 and the molecular mechanisms underlying the pathogenesis of PE in HTR8/SVneo cells. Furthermore, the serum expression of ANGPTL8 and its potential correlation in patients with PE and healthy subjects were explored. The current study results provided evidence that ANGPTL8 affects the proliferation, invasion and migration of trophoblast cells to promote PE.

\section{Materials and methods}

Study subjects and collection of blood samples. A total of 30 patients with $\mathrm{PE}$ and 30 healthy pregnant women who underwent cesarean deliveries and hospitalized (July 2016 to April 2018) in Xuzhou Central Hospital (Xuzhou, China) were recruited into the present study. The main clinicopathological characteristics of the participants are summarized in Table I. All corresponding blood samples were collected after delivery (within $1 \mathrm{~h}$ ) and used according to the protocol approved by the Ethics Committee at Xuzhou Central Hospital. Pregnant women were excluded from the present study if they presented any of the following: $<18$ years of age without a legal guardian, was at $>26$ weeks of gestation, had a multifetal gestation, suffered from a cardiovascular disease (including chronic hypertension or valvular heart disease) or pregestational diabetes, or had a history of fenfluramine/phentermine use. All participants provided written informed consent.

Cell culture. The immortalized human trophoblast cell line HTR8/SVneo was purchased from the Type Culture Collection of the Chinese Academy of Sciences. HTR/SVneo cells were cultured in RPMI 1640 medium supplemented with $10 \%$ fetal bovine serum (FBS; Gibco; Thermo Fisher Scientific, Inc.), $100 \mathrm{U} / \mathrm{ml}$ penicillin and $100 \mu \mathrm{g} / \mathrm{ml}$ streptomycin (Invitrogen; Thermo Fisher Scientific, Inc.). The cells were incubated in a humidified atmosphere containing $95 \%$ air and $5 \% \mathrm{CO}_{2}$ at $37^{\circ} \mathrm{C}$.

Small interfering RNA (siRNA) transfection. siRNAs were synthesized by Santa Cruz Biotechnology, Inc., and transfected into the HTR8/SVneo cells using Lipofectamine 2000 (Invitrogen; Thermo Fisher Scientific, Inc.) according to the manufacturer's protocols. The cells were divided into three groups, as follows: Control group (without any treatment), si-negative control (NC) group (transfected with an unrelated sequence), and si-ANGPTL8 group (transfected with ANGPTL8-siRNA). At $48 \mathrm{~h}$ post-transfection, the cells were harvested and subjected to western blotting or reverse transcription-quantitative polymerase chain reaction (RT-qPCR) assay to detect the efficiency of transfection. The sequences of si-ANGPTL8 used are as follows: si-ANGPTL8-1: 5'-AAG CCCACCAAGAATTTGAGA-3'; si-ANGPTL8-2: 5'-TAT GACAGAGCACTGGAATTC-3'.

Serum ANGPTL8 measurements. Serum was separated by centrifugation $(900 \mathrm{x} \mathrm{g} ; 5 \mathrm{~min})$ of the blood samples at $4^{\circ} \mathrm{C}$ and stored at $-80^{\circ} \mathrm{C}$ until further assay. Subsequently, the serum concentration of ANGPTL8 was quantified using an ELISA kit (cat. no. NBP2-68217; R\&D Systems) according to the manufacturer's protocol.

RT-qPCR assay. Cells were washed with ice-cold phosphate buffer, and total RNA was extracted using TRIzol reagent (Invitrogen; Thermo Fisher Scientific, Inc.), and measured using a Qubit machine (Invitrogen; Thermo Fisher Scientific, Inc.), according to the manufacturer's protocol. Next, RNA $(1 \mu \mathrm{g})$ was reversed transcribed using RT reagents (Takara Bio, Inc.) to synthesize complementary DNA. qPCR was then conducted using a 7500 Real-Time PCR system (Applied Biosystems; Thermo Fisher Scientific, Inc.) and the following PCR cycling protocol was used: $95^{\circ} \mathrm{C}$ for $2 \mathrm{~min}$ and then 40 cycles of $94^{\circ} \mathrm{C}$ for $15 \mathrm{sec}, 60^{\circ} \mathrm{C}$ for $20 \mathrm{sec}, 72^{\circ} \mathrm{C}$ for $20 \mathrm{sec}$, followed by $72^{\circ} \mathrm{C}$ for $7 \mathrm{~min}$. The primer sequences for qPCR were as follows: ANGPTL8 forward, 5'-ATTCCTGGGGAC AGAAGTCA-3', and reverse, 5'-GCTTTACACCTTCGAGCT GA-3'; and GAPDH forward, 5'-CTCACCGGATGCACCAAT GTT-3', and reverse, 5'-CGCGTTGCTCACAATGTTCAT-3'. GAPDH was used as an internal control for mRNA expression, and the relative mRNA expression was calculated by the $2^{-\Delta \Delta C q}$ method (18).

Western blot analysis. After transfection for $24 \mathrm{~h}$, cells were harvested, and total protein was extracted using RIPA lysis buffer (Beyotime Institute of Biotechnology). Protein concentration was then measured with a BCA kit (Thermo Fisher Scientific, Inc.). A total of $25 \mu \mathrm{g}$ protein was separated by SDS-PAGE (10\%), transferred to PVDF membranes (EMD Millipore) and blocked in 5\% non-fat milk for $2 \mathrm{~h}$ at room temperature. The membranes were incubated with the primary antibodies overnight at $4^{\circ} \mathrm{C}$, followed by incubation with HRP-conjugated goat anti-rabbit IgG (cat. no. R2004; 1:5,000; Sigma-Aldrich; Merck KGaA) or goat anti-mouse IgG (cat. no. A6715; 1:5,000; Sigma-Aldrich; Merck KGaA) for $1 \mathrm{~h}$ at room temperature. The antibodies used in the present study were as follows: Anti-ANGPTL8 (1:1,000; cat. no. CSB-PA757793LA01HU), purchased from Cusabio; anti-cyclin-dependent kinase 2 (CDK2; 1:1,000; cat. no. 2546), anti-p21 (1:1,000; cat. no. 2947), anti-proliferating cell nuclear antigen (PCNA; 1:1,000; cat. no. 13110), anti-matrix metalloproteinase (MMP)-2 (1:1,000; cat. no. 40994), anti-MMP-9 (1:1,000; cat. no. 13667), anti-tissue inhibitor of matrix metalloproteinase (TIMP)-1 (1:1,000; cat. no. 8946) and anti-TIMP-2 (1:1,000; cat. no. 5738) antibodies, obtained from Cell Signaling Technology, Inc. The proteins were visualized using a Tanon-5200 Chemiluminescence Imager 
Table I. Baseline characteristics of subjects in the study population ( $\mathrm{n}=30$ per group).

\begin{tabular}{|c|c|c|c|}
\hline Characteristics & Preeclampsia $(n=30)$ & Healthy controls $(n=30)$ & P-value \\
\hline \multicolumn{4}{|l|}{ Maternal age $(\text { years })^{\mathrm{a}}$} \\
\hline$\leq 35$ & $25(83.3 \%)$ & $27(90.0 \%)$ & 0.54 \\
\hline$>35$ & $5(16.7 \%)$ & $3(10.0 \%)$ & \\
\hline Body mass index & $28.3 \pm 1.86(22-32)$ & $25 \pm 1.12(21-29)$ & $<0.001$ \\
\hline \multicolumn{4}{|l|}{ Blood pressure } \\
\hline Systolic & $139 \pm 10(118-160)$ & $115 \pm 10(103-127)$ & $<0.001$ \\
\hline Diastolic & $95 \pm 5(84-100)$ & $78.7 \pm 12.3(65-93)$ & $<0.001$ \\
\hline Proteinuria & $2.5 \pm 0.65(0.5-4.5)$ & $0.13 \pm 0.05(0-0.3)$ & $<0.001$ \\
\hline Gestational age at delivery (years) & $33.3 \pm 1.84(31-35)$ & $33.2 \pm 2.53(31-36)$ & 0.71 \\
\hline Previous pregnancy ${ }^{\mathrm{a}}$ & $14(46.7 \%)$ & $15(50 \%)$ & 0.32 \\
\hline
\end{tabular}

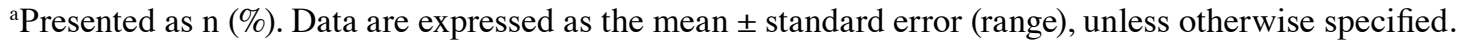

(Tanon Science and Technology Co., Ltd.) and an enhanced chemiluminescence western blotting substrate (EMD Millipore).

Cell proliferation assay. A Cell Counting Kit-8 (CCK-8) assay (Dojindo Molecular Technologies, Inc.) was performed to detect cell viability. Briefly, HTR8/SVneo cells were seeded into 96 -well plates $\left(5 \times 10^{3}\right.$ cells/well $)$ and incubated at $37^{\circ} \mathrm{C}$ with $5 \% \mathrm{CO}_{2}$. At the indicated time points $(24,48$ and $72 \mathrm{~h}$ ) after transfection, $10 \mu \mathrm{l}$ CCK-8 solution was added into each well and incubated for $2 \mathrm{~h}$ at $37^{\circ} \mathrm{C}$ in the dark. The absorbance of each well was then measured with a microplate reader (ELx808; BioTek Instruments, Inc.) at $450 \mathrm{~nm}$.

Wound healing assay. A wound healing assay was performed to detect the migration ability of HTR8/SVneo cells. Briefly, cells $\left(4 \times 10^{4}\right)$ were cultured in six-well plates for $24 \mathrm{~h}$. The cells were transfected with si-NC or si-ANGPTL8-1 for $72 \mathrm{~h}$. HTR8/SVneo cells without treatment served as a control. The monolayers were then scratched vertically using a $200-\mu 1$ sterile pipette tip, and any floating cells were washed off with PBS. Cells were photographed at the indicated time points (0 and $24 \mathrm{~h}$ ) using a Leica DM IL LED microscope equipped with an Integrated 5.0 Mega-Pixel MC170 HD camera (Leica Microsystems $\mathrm{GmbH}$ ).

Transwell invasion assay. A Transwell assay was performed to evaluate the invasive capability of HTR8/SVneo cells with an $8-\mu \mathrm{m}$ pore polycarbonate membrane chamber insert in a 24-well plate (Corning, Inc.). For the invasion assays, the chamber inserts were coated with $50 \mu \mathrm{l}$ Matrigel $(200 \mathrm{mg} / \mathrm{ml}$; BD Biosciences). Briefly, at $24 \mathrm{~h}$ post-transfection, cells were resuspended in $200 \mu \mathrm{l}$ serum-free medium and seeded in the upper chamber of the transwell inserts. A total of $600 \mu 1$ RPMI 1640 medium containing 10\% FBS was added to the lower chambers. After $24 \mathrm{~h}$ of incubation, the non-invading cells were gently removed with a cotton swab, and the invasive cells attached to the lower surface of the chamber membranes were fixed with polyoxymethylene and stained with $0.1 \%$ crystal violet solution. Finally, the number of cells in five random fields was counted, and images were captured under an inverted microscope (Olympus Corporation).

Statistical analysis. The data are presented as the mean \pm standard error of the mean. Differences among the data were analyzed using Student's t-test (unpaired) or analysis of variance followed by Bonferroni correction, as appropriate. Statistical data were analyzed using SPSS software, version 13.0 (SPSS, Inc.). $\mathrm{P}<0.05$ was considered to indicate a statistically significant difference.

\section{Results}

ANGPTL8 expression and secretion are increased in PE. As shown in Table I, there was no significant difference in the age or ratio of previous pregnancy between the pregnant women with $(n=30)$ or without PE $(n=30)$ enrolled in the present study. However, the systolic and diastolic pressure values of pregnant women with PE were higher compared with those exhibited by healthy pregnant women. Next, the ANGPTL8 levels in the serum of the subjects were detected by ELISA. The results revealed that the expression levels of ANGPTL8 were notably increased in pregnant women with PE compared with those observed in the normal controls (Fig. 1A). Correlation analysis further revealed a positive association between the expression level of ANGPTL8 and the body mass index, systolic pressure, diastolic pressure and proteinuria. Based on the results shown in Fig. 1, it can be hypothesized that ANGPTL8 has a potential effect on the pathogenesis of PE.

Downregulation of ANGPTL8 promotes trophoblast cell proliferation. Successful interference on ANGPTL8 expression in trophoblast cells by siRNA transfection was confirmed by western blotting and RT-qPCR. The results demonstrated that the silencing effect of si-ANGPTL8-1 was better than that of si-ANGPTL8-2; thus, si-ANGPTL8-1 was selected for use in subsequent experiments (Fig. 2A and B). Next, a CCK-8 assay was performed to elucidate the function of ANGPTL8 in trophoblast cells, and the results revealed that reduced 
A
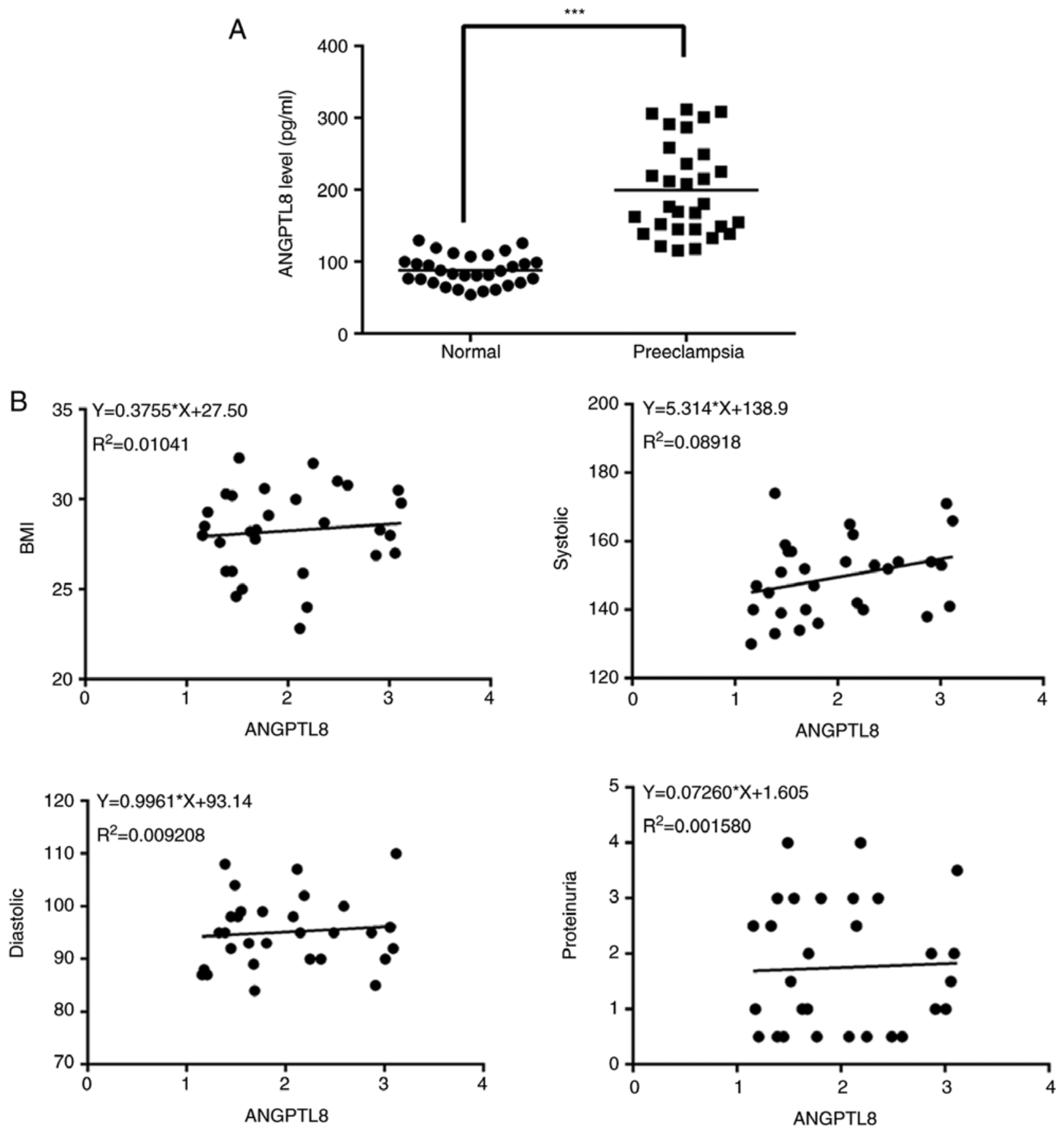

Figure 1. ANGPTL8 expression levels in preeclampsia patients and correlation analysis with baseline characteristics. (A) Levels of ANGPTL8 in the serum of the study population. (B) Correlation analysis of body mass index (BMI), systolic pressure, diastolic pressure and proteinuria with ANGPTL8 levels. ${ }^{* * *} \mathrm{P}<0.001$ vs. control subjects. ANGPTL8, angiopoietin-like protein 8.

expression of ANGPTL8 increased the viability and proliferation of HTR8/SVneo cells (Fig. 2C). Western blotting was also performed to detect the levels of proteins involved in cell proliferation, including CDK2, p21 and PCNA. As shown in Fig. 2D, downregulation of ANGPTL8 decreased p21 expression, and increased the CDK2 and PCNA expression levels in HTR8/SVneo cells compared with the expression levels in the control and si-NC groups.

Downregulation of ANGPTL8 promotes trophoblast cell migration and invasion. Wound healing and transwell assays were performed to investigate the effect of ANGPTL8 on trophoblast cell migration and invasion, respectively. The results demonstrated that silencing of ANGPTL8 led to a significant acceleration in the migratory and invasive capacities of trophoblast cells (Fig. 3A and B). Next, western blotting was used to evaluate the levels of proteins associated with migration and invasion, including MMP-2, MMP-9, TIMP-1 and TIMP-2. The data revealed that downregulation of ANGPTL8 markedly enhanced the levels of MMP-2 and MMP-9, while the protein expression levels of TIMP-1 and TIMP-2 were significantly decreased in the si-ANGPTL8-1 group as compared with those in the control and si-NC groups (Fig. 3C). 
A

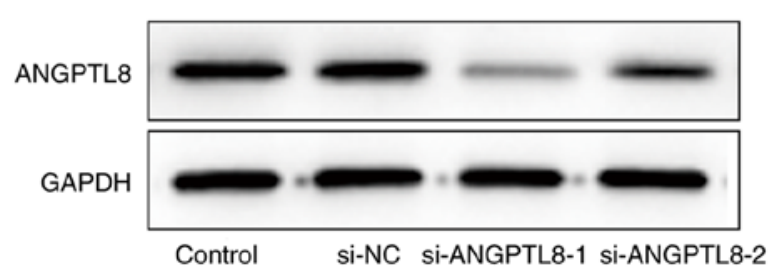

B

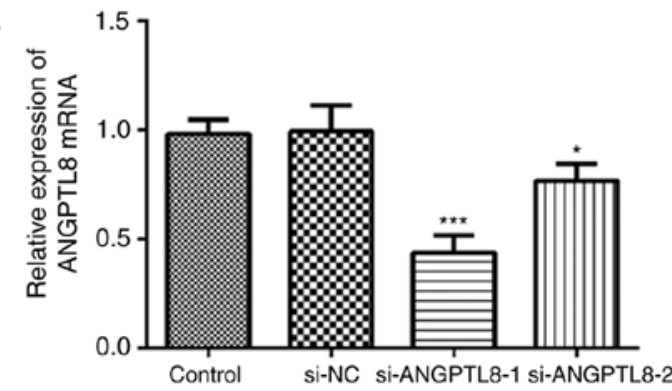

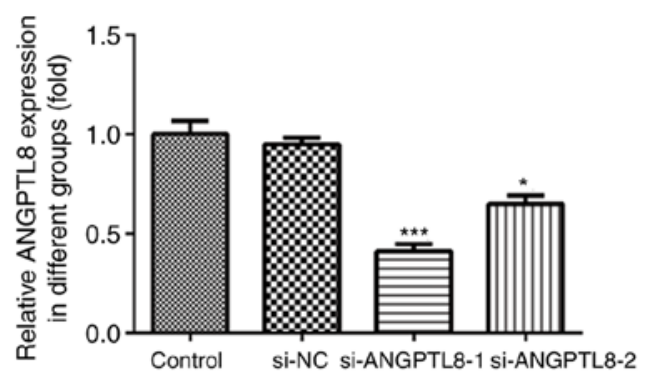

C

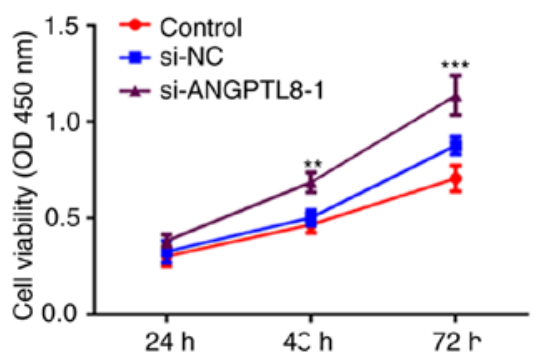

D
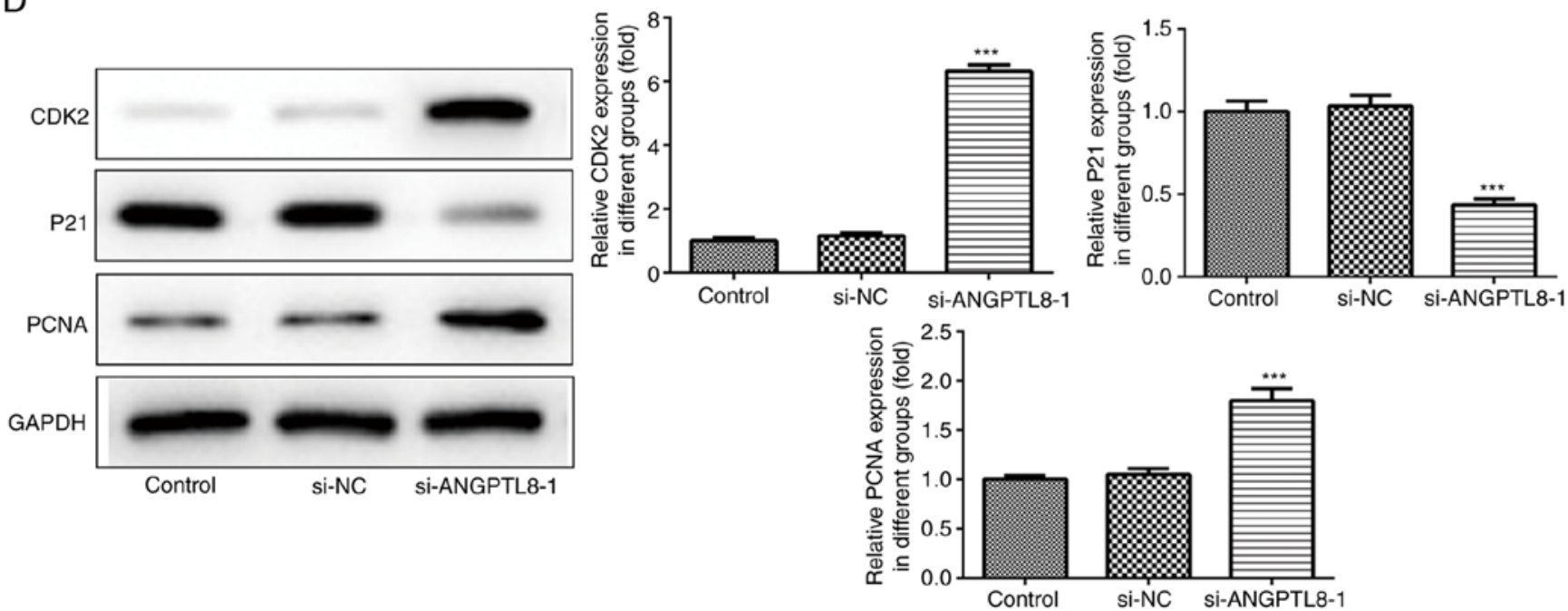

Figure 2. Effect of ANGPTL8 on the viability and proliferation of trophoblast cells. HTR8/SVneo cells were transfected with si-NC or si-ANGPTL8. (A) Western blotting and (B) reverse transcription-quantitative polymerase chain reaction were performed to evaluate the protein and mRNA expression of ANGPTL8, respectively. (C) Cell Counting Kit-8 assay was used to detect the viability of HTR8/SVneo cells. (D) The expression levels of CDK2, p21 and PCNA were analyzed by western blotting. The data represent the mean \pm standard error of the mean of three independent experiments. ${ }^{*} \mathrm{P}<0.05,{ }^{* *} \mathrm{P}<0.01$ and ${ }^{* * *} \mathrm{P}<0.001$, vs. control or si-NC group. ANGPTL8, angiopoietin-like protein 8; si-, small interfering RNA; NC, negative control; CDK2, cyclin-dependent kinase 2; PCNA, proliferating cell nuclear antigen.

\section{Discussion}

$\mathrm{PE}$ is a systemic disorder that occurs during pregnancy and is characterized by various manifestations of organ dysfunction. PE causes gestational hypertension and proteinuria, which is a sign of renal dysfunction (19). The clinical data reported in the present study indicated that the systolic and diastolic pressure values of pregnant women with PE were higher compared with those of healthy pregnant women, as shown in Table I. PE is considered as a two-stage disorder, since the pathogenesis and mechanisms of PE are considered to involve antiangiogenic and angiogenic factors and/or placental debris, leading to trophoblast cell apoptosis and abnormal development of the placenta (20-22). A previous study reported that PE is an ischemic placental disease, associated with trophoblastic metabolic disorders (23). Defective trophoblast invasion of the uterus has been reported to be one of the crucial mechanisms of PE. Furthermore, increased oxidative stress and reduced uteroplacental blood flow and oxygen availability were closely associated with reduced trophoblast invasion (24).

ANGPTL8 is a newly identified hormone with the ability to regulate the glucose and lipid metabolic pathways. Abnormal expression of ANGPTL8 has been reported in nonalcoholic fatty liver disease, insulin resistance and diabetes mellitus (25). In addition, ANGPTL8 exerts a protective effect on atherosclerosis through the inhibition of HDL-mediated cholesterol efflux capacity (26). A previous study demonstrated that ANGPTL8 levels were increased in obesity, impaired glycometabolism and dyslipidemia (13). In the present study, the results revealed that ANGPTL8 expression was also increased in pregnant women with PE as 
A
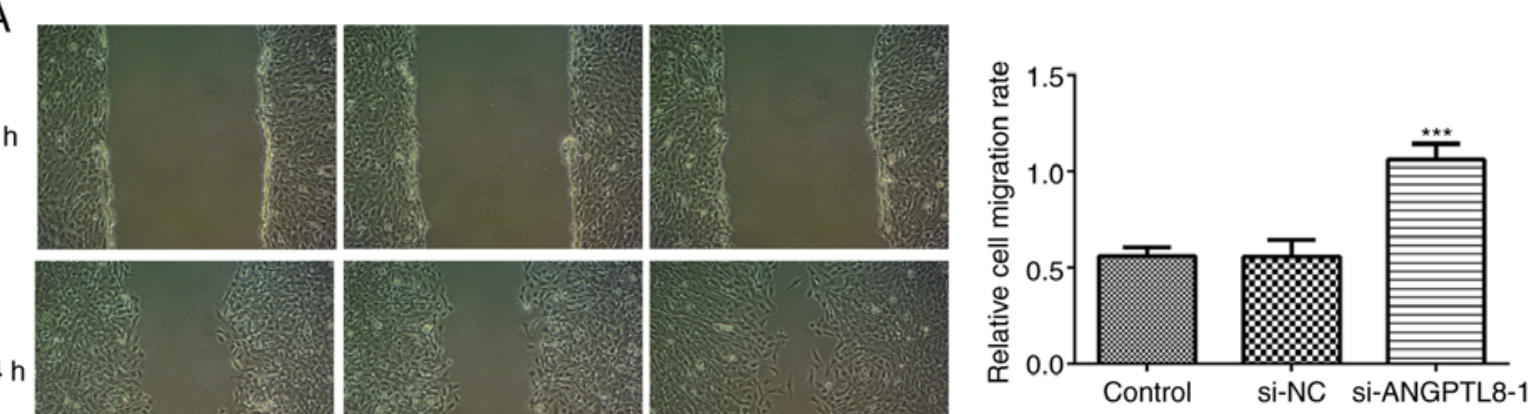

$24 \mathrm{~h}$

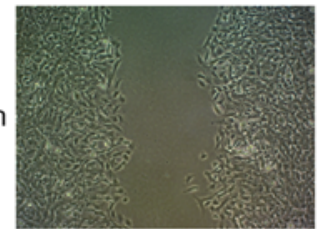

Control

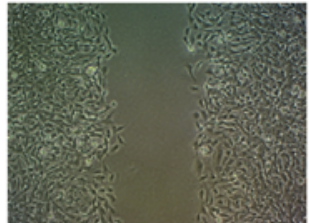

si-NC

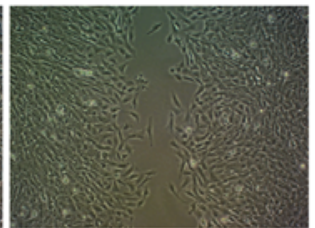

si-ANGPTL8-1

B

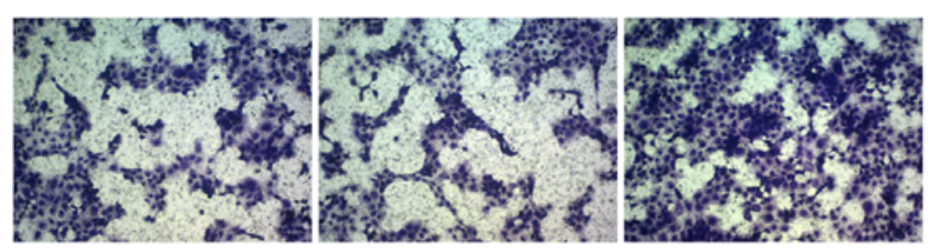

Control

si-ANGPTL8-1
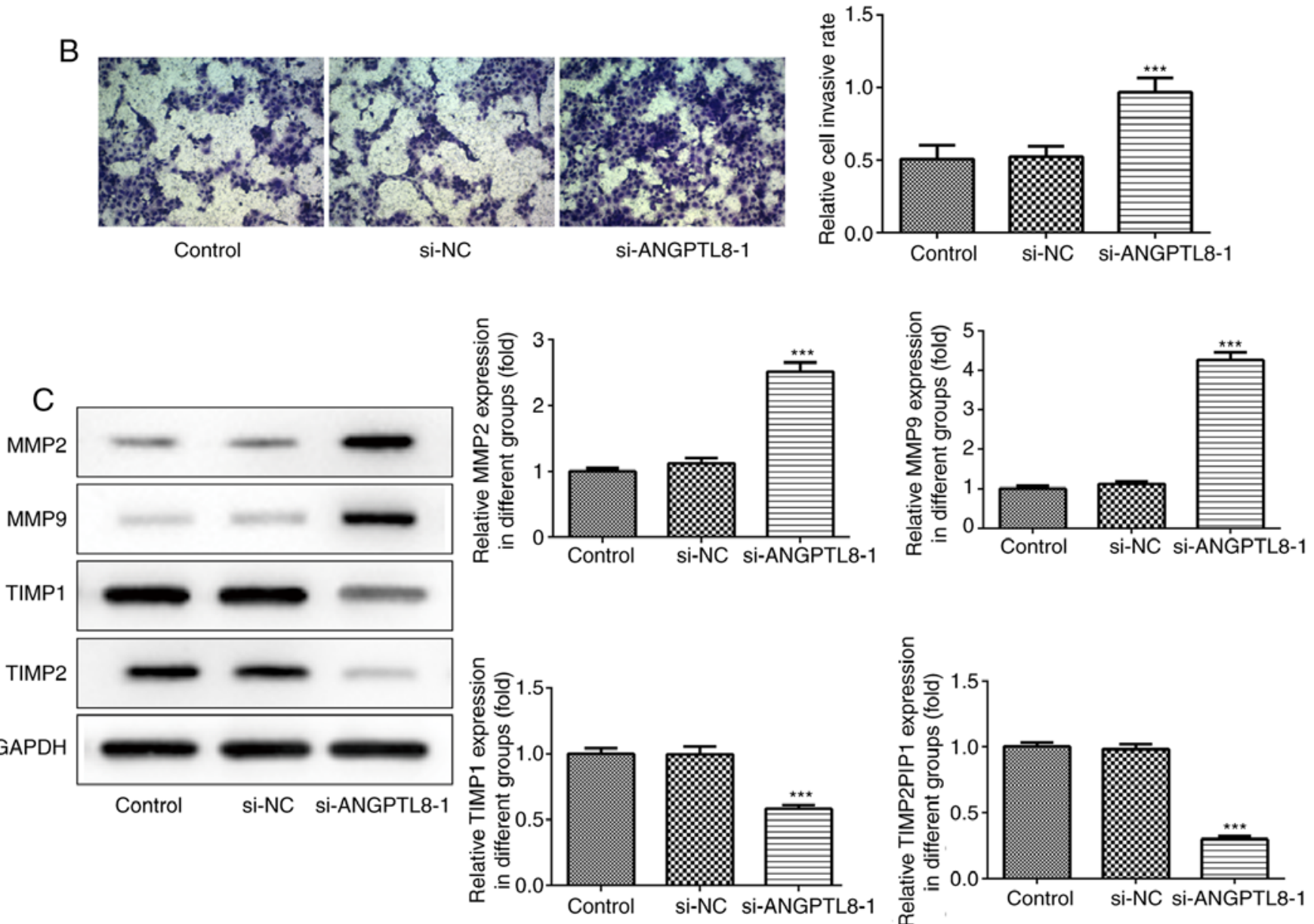

Figure 3. Effect of ANGPTL8 on the migration and invasion of trophoblast cells. (A) Wound healing assay and (B) Transwell assay were performed to investigate the effect of ANGPTL8 on cell migration and invasion, respectively (magnification, x100). (C) The effect of ANGPTL8 on the protein expression levels of MMP-2, MMP-9, TIMP-1 and TIMP-2 was analyzed by western blotting. The data represent the mean \pm standard error of the mean of three independent experiments. ${ }^{* * *} \mathrm{P}<0.001$ vs. control or si-NC group. ANGPTL8, angiopoietin-like protein 8; si-, small interfering RNA; NC, negative control; MMP, matrix metalloproteinase; TIMP, tissue inhibitor of matrix metalloproteinase.

compared with that in the normal controls (Fig. 2). Therefore, it was hypothesized that ANGPTL8 is involved in the pathogenesis of PE.

The current study further employed HTR8/SVneo cells to investigate the aberrant trophoblastic invasion in the pathogenesis of $\mathrm{PE}$ in vitro. The HTR8/SVneo cells were transfected with ANGPTL8-siRNA to downregulate ANGPTL8 expression in order to investigate the molecular mechanism by which ANGPTL8 regulates trophoblast invasion during PE development. It is widely accepted that excessive apoptosis and poor invasion of trophoblast cells serve major roles in the pathogenesis of PE. Wang et al reported that regulation of the microRNA-210/NOTCH1 signaling pathway attenuated the impaired HTR-8/SVneo cell proliferation, migration and invasion, which contributed to trophoblast dysfunction in the placenta of patients with PE (27). This is consistent with the data of the present study, which indicated that downregulation of ANGPTL8 increased the viability and proliferation of HTR8/SVneo cells (Fig. 2C), as well as corrected for the expression levels of 
proteins involved in cell proliferation, including CDK2, p21 and PCNA. Furthermore, silencing of ANGPTL8 promoted trophoblast cell migration and invasion, and altered the expression levels of proteins associated with migration and invasion, including MMP-2, MMP-9, TIMP-1 and TIMP-2 (Fig. 3). Wu et al (28) previously demonstrated that downregulation of hypoxia-inducible factor $1 \alpha$ antisense RNA-2 may be involved in the pathogenesis and progression of PE by decreasing trophoblast cell proliferation, migration and invasion. Furthermore, Ebegboni et al (29) reported that a dietary intake of food that is rich in quercetin or hesperidin during early pregnancy was able to significantly improve trophoblast (placenta) health and function against oxidative stress by enhancing spheroid stem-like cell generation in HTR8/SVneo cells, thus aiding their invasion (29). These previous findings, along with the results of the present study, provide an insight into the potential role of ANGPTL8 in the process of $\mathrm{PE}$.

In conclusion, the present study demonstrated that ANGPTL8 serves an important role in the pathogenesis of $\mathrm{PE}$, and that the downregulation of ANGPTL8 significantly promoted trophoblast cell proliferation, invasion and migration. Collectively, these data support the potential application of ANGPTL8 as a target for clinical diagnosis and treatment of PE.

\section{Acknowledgements}

Not applicable.

\section{Funding}

No funding was received.

\section{Availability of data and materials}

The datasets used and/or analyzed during the present study are available from the corresponding author on reasonable request.

\section{Authors' contributions}

WW and XL designed the experiments and drafted the manuscript. DJ mainly performed the experiments and analyzed the data. WW performed the western blot and RT-qPCR experiments. XL reviewed the manuscript. All authors read and approved the final manuscript.

\section{Ethics approval and consent to participate}

The present study was approved by the Ethics Committee of Xuzhou Central Hospital (Xuzhou, China). All participants provided written informed consent.

\section{Patient consent for publication}

All participants provided written informed consent for publication.

\section{Competing interests}

The authors declare that they have no competing interests.

\section{References}

1. Steegers EA, von Dadelszen P, Duvekot JJ and Pijnenborg R: Pre-eclampsia. Lancet 376: 631-644, 2010.

2. Bilano VL, Ota E, Ganchimeg T, Mori R and Souza JP: Risk factors of pre-eclampsia/eclampsia and its adverse outcomes in low- and middle-income countries: A WHO secondary analysis. PLoS One 9: e91198, 2014.

3. Ospina-Prieto S, Chaiwangyen W, Herrmann J, Groten T, Schleussner E, Markert UR and Morales-Prieto DM: MicroRNA-141 is upregulated in preeclamptic placentae and regulates trophoblast invasion and intercellular communication. Transl Res 172: 61-72, 2016.

4. Lenfant C: Working group report on high blood pressure in pregnancy. J Clin Hyper (Greenwich) 3: 75-88, 2001.

5. Wu P, Haththotuwa R, Kwok CS, Babu A, Kotronias RA, Rushton C, Zaman A, Fryer AA, Kadam U, Chew-Graham CA and Mamas MA: Preeclampsia and future cardiovascular health: A systematic review and meta-analysis. Circ Cardiovasc Qual Outcomes 10: e003497, 2017.

6. He P, Chen Z, Sun Q, Li Y, Gu H and Ni X: Reduced expression of $11 \beta$-hydroxysteroid dehydrogenase type 2 in preeclamptic placentas is associate $\beta \mathrm{d}$ with decreased PPAR $\gamma$ but increased PPAR $\alpha$ expression. Endocrinology 155: 299-309, 2014.

7. Yu L, Li D, Liao QP, Yang HX, Cao B, Fu G, Ye G, Bai Y, Wang H, Cui N, et al: High levels of activin A detected in preeclamptic placenta induce trophoblast cell apoptosis by promoting nodal signaling. J Clin Endocrinol Metab 97: E1370-E1379, 2012.

8. Wallace AE, Fraser R, Gurung S, Goulwara SS, Whitley GS Johnstone AP and Cartwright JE: Increased angiogenic factor secretion by decidual natural killer cells from pregnancies with high uterine artery resistance alters trophoblast function. Hum Reprod 29: 652-660, 2014.

9. Wallace AE, Whitley GS, Thilaganathan B and Cartwright JE: Decidual natural killer cell receptor expression is altered in pregnancies with impaired vascular remodeling and a higher risk of pre-eclampsia. J Leukoc Biol 97: 79-86, 2015.

10. Wang Y, Hao M, Sampson S and Xia J: Elective delivery versus expectant management for pre-eclampsia: A meta-analysis of RCTs. Arch Gynecol Obstet 295: 607-622, 2017.

11. Kersten S: Physiological regulation of lipoprotein lipase. Biochim Biophys Acta 1841: 919-933, 2014.

12. Quagliarini F, Wang Y, Kozlitina J, Grishin NV, Hyde R, Boerwinkle E, Valenzuela DM, Murphy AJ, Cohen JC and Hobbs HH: Atypical angiopoietin-like protein that regulates ANGPTL3. Proc Natl Acad Sci USA 109: 19751-19756, 2012.

13. Morinaga J, Zhao J, Endo M, Kadomatsu T, Miyata K, Sugizaki T, Okadome Y, Tian Z, Horiguchi H, Miyashita K, et al: Association of circulating ANGPTL 3,4 , and 8 levels with medical status in a population undergoing routine medical checkups: A cross-sectional study. PLoS One 13: e0193731, 2018.

14. Lee EC, Desai U, Gololobov G, Hong S, Feng X, Yu XC, Gay J, Wilganowski N, Gao C, Du LL, et al: Identification of a new functional domain in angiopoietin-like 3 (ANGPTL3) and angiopoietin-like 4 (ANGPTL4) involved in binding and inhibition of lipoprotein lipase (LPL). J Biol Chem 284: 13735-13745, 2009.

15. Yoshida K, Shimizugawa $T$, Ono $M$ and Furukawa $\mathrm{H}$ : Angiopoietin-like protein 4 is a potent hyperlipidemia-inducing factor in mice and inhibitor of lipoprotein lipase. J Lipid Res 43: 1770-1772, 2002

16. Liu L, Zhuang X, Jiang M, Guan F, Fu Q and Lin J: ANGPTL4 mediates the protective role of PPARgamma activators in the pathogenesis of preeclampsia. Cell Death Dis 8: e3054, 2017.

17. Luo M and Peng D: ANGPTL8: An important regulator in metabolic disorders. Front Endocrinol (Lausanne) 9: 169, 2018.

18. Livak KJ and Schmittgen TD: Analysis of relative gene expression data using real-time quantitative PCR and the 2(-Delta Delta C(T)) method. Methods 25: 402-408, 2001.

19. Brown MA, Magee LA, Kenny LC, Karumanchi SA, McCarthy FP, Saito S, Hall DR, Warren CE, Adoyi G and Ishaku S; International Society for the Study of Hypertension in Pregnancy (ISSHP): The hypertensive disorders of pregnancy: ISSHP classification, diagnosis \& management recommendations for international practice. Pregnancy Hypertens 13: 291-310, 2018.

20. Chelbi ST and Vaiman D: Genetic and epigenetic factors contribute to the onset of preeclampsia. Mol Cell Endocrinol 282: 120-129, 2008.

21. Seki H: Balance of antiangiogenic and angiogenic factors in the context of the etiology of preeclampsia. Acta Obstet Gynecol Scand 93: 959-964, 2014. 
22. Shah DA and Khalil RA: Bioactive factors in uteroplacental and systemic circulation link placental ischemia to generalized vascular dysfunction in hypertensive pregnancy and preeclampsia. Biochem Pharmacol 95: 211-226, 2015.

23. Makris A, Yeung KR, Lim SM, Sunderland N, Heffernan S, Thompson JF, Iliopoulos J, Killingsworth MC, Yong J, $\mathrm{Xu} \mathrm{B}$, et al: Placental growth factor reduces blood pressure in a uteroplacental ischemia model of preeclampsia in nonhuman primates. Hypertension 67: 1263-1272, 2016.

24. Gutiérrez JA, Gómez I, Chiarello DI, Salsoso R, Klein AD, Guzmán-Gutiérrez E, Toledo F and Sobrevia L: Role of proteases in dysfunctional placental vascular remodelling in preeclampsia. Biochim Biophys Acta Mol Basis Dis 1866: 165448, 2020.

25. Siddiqa A, Cirillo E, Tareen SHK, Ali A, Kutmon M, Eijssen LMT, Ahmad J, Evelo CT and Coort SL: Biological pathways leading from ANGPTL8 to diabetes mellitus-A co-expression network based analysis. Front Physiol 9: 1841, 2018.

26. Luo M, Zhang Z, Peng Y, Wang S and Peng D: The negative effect of ANGPTL8 on HDL-mediated cholesterol efflux capacity. Cardiovasc Diabetol 17: 142, 2018.
27. Wang R, Liu W, Liu X, Liu X, Tao H, Wu D, Zhao Y and Zou L: MicroRNA-210 regulates human trophoblast cell line HTR-8/SVneo function by attenuating Notch1 expression: Implications for the role of microRNA-210 in pre-eclampsia. Mol Reprod Dev 86: 896-907, 2019.

28. Wu D, Yang N, Xu Y, Wang S, Zhang Y, Sagnelli M, Hui B, Huang $Z$ and Sun L: lncRNA HIF1A antisense RNA 2 modulates trophoblast cell invasion and proliferation through upregulating PHLDA1 expression. Mol Ther Nucleic Acids 16: 605-615, 2019.

29. Ebegboni VJ, Dickenson JM and Sivasubramaniam SD: Antioxidative effects of flavonoids and their metabolites against hypoxia/reoxygenation-induced oxidative stress in a human first trimester trophoblast cell line. Food Chem 272: 117-125, 2019.

This work is licensed under a Creative Commons Attribution-NonCommercial-NoDerivatives 4.0 International (CC BY-NC-ND 4.0) License. 